\title{
Aprendendo com o Passado: Opções Urgentes para a Prevenção e Tratamento de Infeções Respiratórias Agudas Severas Causadas pelo Vírus SARS-CoV-2
}

A trajetória atual do surto de 2019-nCoV (COVID-19) causado pelo vírus SARS-CoV-2, tornou necessário o desenvolvimento de medidas de saúde pública para conter a disseminação do vírus e a otimização dos tratamentos dos doentes infetados. Uma análise da sequência do genoma do SARS-CoV-2, disponível em GenBank (ID: MN908947.3), mostra uma forte homologia com o SARS-CoV. Portanto, os estudos previamente desenvolvidos sobre o SARS-CoV e a Síndrome Respiratória no Oriente Médio (MERS-CoV) podem ser usados diretamente para ajudar a tratar a COVID-19.

Wenshe Ray Liu e o seu grupo de investigação (Universidade A\&M do Texas) concentraram os seus esforços no sentido de encontrar medicamentos para tratar a COVID-19.

Partindo de um pequeno conjunto de moléculas (peptídeo baseado em ACE2, remdesivir, 3CLpro-1 e novas vinilsulfonas inibidores de protease), o grupo de Liu identificou a droga antiviral remdesivir (desenvolvido para dar resposta à pandemia de Ébola em 2014) como um medicamento viável para tratar a COVID-19. No estudo, publicado no final de janeiro, os autores identificam ainda outras "pequenas-moléculas" com potencial terapêutico para a COVID-19, como por exemplo o 3CLpro-1.

$>$

Marta Piñeiro

mpineiro@qui.uc.pt<smiles>CCC(CC)COC(=O)[C@H](C)NP(=O)(OCC1C[C@@](C#N)(c2ccc3c(N)ncnn23)[C@H](O)[C@@H]1O)Oc1ccccc1</smiles>

remdesivir<smiles>O=C[C@H](C[C@@H]1CCNC1=O)NC(=O)[C@H](Cc1ccccc1)NC(=O)/C=C/c1ccc(Cl)cc1F</smiles>

3CLpro-1

\section{Fontes}

Chemists working on drugs to treat COVID-19, ScienceDaily. ScienceDaily, 6 Abril 2020. www.sciencedaily.com/releases/2020/04/200406190509.htm.

J. S. Morse, T. Lalonde, S. Xu, W. R. Liu, ChemBioChem 21 (2020) 730-738. DOI: $10.1002 /$ cbic.202000047 